\title{
Treatment Outcomes of Patients with Superior Vena Cava Syndrome who Received High Dose Radiotherapy at UP - PGH from 2009- 2011
}

\author{
Maria Teresa U. Benedicto, Jubert P. Benedicto, Marbe Joy A. Depayso and Elaine Johana C. Limkin
}

Section of Radiation Oncology, Department of Radiology, College of Medicine and Philippine General Hospital, University of the Philippines Manila

\begin{abstract}
Background. 85\% of cases of Superior Vena Cava Obstruction (SVCO) are caused by malignancy. Radiation therapy (RT) is an effective therapeutic modality for SVCO localized in the thorax, especially for obstructive causes.
\end{abstract}

Objective. To determine the clinical profile and outcomes of patients who received radiation treatment at UP-PGH from January 1, 2009-December 31, 2011.

Methods. Records of patients with SVCO were reviewed. The patient's demographic profiles were collected. Outcomes were labeled as recovered, improved, not improved and died based on standard definitions.

Study Design. Retrospective, descriptive study.

Study Setting. Philippine General Hospital

Study Population. UP-PGH patients with SVCO from 2009 - 2011.

Results. The majority of the referrals were from general medicine (50\%) and the most common symptom was dyspnea (73\%); however, it took the patients $2-4$ weeks from the onset of symptoms to seek consult. $36 \%$ of patients had lung adenocarcinoma with disseminated disease at diagnosis. The majority $(73 \%)$ showed symptomatic response after receiving the SVC dose.

Conclusion. The importance of a multidisciplinary approach to patient care cannot be overemphasized. Improvement in signs and symptoms, particularly with that of dyspnea, was noted in $72.5 \%$ of patients after receiving radiation therapy. Of the 8 patients who followed up for completion therapy, $5 / 8$ of them reported significant improvement.

Key Words: SVC Syndrome, obstruction, outcome, radiotherapy

Corresponding author: Ma Teresa U. Benedicto, MD

Section of Radiation Oncology

Department of Radiology

Philippine General Hospital

University of the Philippines Manila

Taft Avenue, Ermita, Manila 1000 Philippines

Telephone: +6325548471

Fax: +6325226597

Email: benedicto_md@yahoo.com
Introduction

\section{Background}

Studies have shown that the presence of SVCO does not imply a poor prognosis in itself. The major factor determining survival is the underlying disease, not the presence of the syndrome, which can be corrected with therapy. At UP-Philippine General Hospital, more than 85\% of cases presenting with clinical evidence of SVCO are due to malignant causes. In our local setting where resources and diagnostic tests are limited, patients' options are usually confined to chemotherapy and/or radiotherapy. Prompt initiation of the appropriate treatment may afford relief of symptoms and improvement in the quality of life which is the major goal in the overall management of these patients.

\section{Rationale}

UP-PGH is a tertiary referral hospital in which the majority of patients seen will already have undergone extensive diagnostic procedures at other institutions. These patients usually present with late complications of their conditions. Among those with SVCO on presentation, one of the management options is palliative radiotherapy once histopathologic results are available. This study will provide a clinical profile of our patients and evaluation of their treatment outcomes. In addition, it may also provide the assigned physicians insights to better delivery of healthcare and follow-up of these patients with possible emphasis on the time elements involved in the referral process.

\section{Objectives}

1. To determine outcomes of patients after receiving palliative radiotherapy.

2. To describe the demographic data and underlying disease conditions of patients who presented with SVCO who received radiation therapy at the UPPGH

\section{Review of Related Literature}

The superior vena cava is the major drainage system for blood returning from the upper extremities, head and neck. It is a thin-walled vessel measuring $6-8 \mathrm{~cm}$ in length and $1.5-2.0 \mathrm{~cm}$ in width lies within a nondistensible space in the mediastinum, making it susceptible to compression. ${ }^{1}$ The 
increased venous pressure in the upper body during compression results in facial plethora, edema of the head, neck and arms, cyanosis, and distended subcutaneous vessels. The edema in turn may cause functional compromise of the nearby structures manifesting as hoarseness of voice, dysphagia, dyspnea and stridor. Dyspnea is the most common presenting symptom of SVCO. ${ }^{1}$ Before the advent of antibiotic therapy, most cases of SVCO were due to infectious etiologies, especially syphilitic aortic aneurysm and tuberculosis. ${ }^{2}$ Today, malignant causes represent $85 \%$ to $97 \%$ of all cases. ${ }^{3}$ A review of 125 cases by Armstrong et al. showed that pulmonary tumors were responsible for $80 \%$ of the SVCO, largely due to non small cell lung cancer followed by thoracic lymphomas. ${ }^{4}$

Diagnosis of SVCO is made on the basis of clinical signs and symptoms. The most useful imaging strategy is computed tomography scan of the chest with intravenous contrast. This modality, combined with a good history, helps in differentiating between vena caval thrombosis and extrinsic compression. It may also help in guiding attempts with biopsy by mediastinoscopy, bronchoscopy, or fine needle aspiration biopsy. Magnetic resonance imaging may be useful for patients who cannot tolerate the contrast medium. A venography may be warranted only when an intervention (placement of a stent or surgery) is planned. ${ }^{5}$

Treatment options for SVCO due to malignant tumors include chemotherapy, radiation therapy, thrombolysis, stent placement and surgery. Chemotherapy is best for lymphoma and small cell cancer. Rapid initiation can result in complete and partial response rates of SVCO in more than $80 \%$ of small cell lung cancer patients, thus its presence should not change the treatment approach. ${ }^{6}$ Radiation therapy is initiated among patients with residual tumors or tumors that are not sensitive to chemotherapy. Its emergent use in hemodynamically stable patients is not of utmost priority for it can obscure histologic diagnosis. Treatment without an established diagnosis should be limited to those patients with rapidly progressive symptoms or those in whom repeated biopsy attempts have been unsuccessful.

If radiation is given as the initial treatment, the field should encompass gross disease and the adjacent nodal regions, taking into consideration the volume of pulmonary, cardiac, and neurologic tissue included in the radiation fields to minimize complications. Many fractionation schemes have been used depending on the diagnosis. Recommended doses for lymphomas are daily fractions of 1.8 to $2.0 \mathrm{~Gy}^{6}$ and 2.0 to $3.0 \mathrm{~Gy}$ for small cell and non small cell lung cancer. Relief of symptoms in small cell lung cancer is reported to be $62-80 \%$, while in non- small cell lung cancer, approximately $46 \%$ of the patients experienced symptomatic relief. ${ }^{7,8}$ In one study, more than $90 \%$ of the patients achieved a partial or complete response with a 3week regimen of $8 \mathrm{~Gy}$ given once a week for a total dose of 24 Gy. ${ }^{9}$ The size and configuration of the field may be altered after the administration of several fractions, as symptoms begin to subside and the staging and plans for subsequent management are organized. Another study has shown that a total dose of $20 \mathrm{~Gy}$ in five fractions or $30 \mathrm{~Gy}$ in 10 fractions is usually adequate for palliation. ${ }^{10}$

If the SVCO is due to a partially occluded vein due to a thrombus, treatment with thrombectomy, thrombolysis or percutaneous placement of an intravascular stent may be warranted. It is particularly an advantage for patients without a tissue diagnosis and are severely symptomatic at presentation. Symptoms such as cyanosis and edema resolve within 48 to 72 hours in most series. ${ }^{11}$ Surgery on an obstructed SVC is infrequently used, and is more appropriate for patients with a benign obstruction than with a malignant obstruction. ${ }^{12}$ It is to note that the severity upon presentation is important in determining the urgency of intervention. In most cases, manifestations are not noticed until the onset of dyspnea; thus, treatment without an established diagnosis may be initiated. ${ }^{1}$ Patients with acute symptoms on admission may benefit from oxygen support, analgesia, elevation of the head of bed, diuretics and corticosteroids. ${ }^{13}$

Radiotherapy plays an important role in palliative medicine.It is usually delivered as a single or a small number of large-dose fractions in a minimal number of hospital visits, in contrast with a large number of small doses in curative radiotherapy. ${ }^{14}$ It can reduce or restrain growth of tumour, preserve function and body image, prevent bony fractures, prevent erosion or compression of blood vessels and abolishes or minimizes symptoms.

The UP-PGH Section of Radiation Oncology, under the Department of Radiology, receives patients with acute symptoms due to SVCO for co-management and palliative radiotherapy. It gets an average of 10 referrals a day, from both the private and the charity sections. The radiation oncologist will assess and take into account the characteristics of the tumor, its location, the extent of disease spread, general health of the patient, co morbid conditions, and other treatment regimens being employed. The best course of radiotherapy will be discussed with the patient and initiated once consent is obtained.

In this institution, radiotherapy is usually given in four 300 cGy fractions or three 400 cGy fractions, both regimens are to reach a total dose of 1200 cGy (so called SVC dose). The chest wall is irradiated in opposing anteroposterior and posteroanterior fields, with the borders dependent on the extent of the mass as seen on imaging. Depending on the histopathologic result obtained from a biopsy of the lesion, the patients will then go on to receive the definitive curative dose of radiation as applicable. For primary lung carcinomas, 180 or 200 cGy daily fractions are given to reach a total dose of 60 to $66 \mathrm{~Gy}$, with or without chemotherapy. For thoracic lymphomas, radiation therapy is commonly given as a consolidation treatment after completion of 
chemotherapy. One hundred eighty or 200 cGy daily fractions are given to reach a total dose of 36 to $45 \mathrm{~Gy}$, depending on the response of the mass to chemotherapy.

An acute side effect of treatment may be the initial radiation edema from high doses of irradiation which could further compromise the respiratory reserve and might lead to an initial worsening of symptoms or sudden death. This can be controlled by giving steroids while on radiotherapy. Side effects of irradiation are usually confined to the organs at risk adjacent to the tumor, such as normal lung parenchyma, spinal cord, esophagus, heart and pericardium. In cases of SVC syndrome, no long term effects are expected as the dose given is below the tolerances of the critical structures mentioned.

\section{Methods}

Study design

Retrospective, descriptive study

\section{Study setting}

Philippine General Hospital - This protocol was reviewed by the hospital's technical review board and approved by the ethics review board of the same institution.

\section{Study population}

\section{Inclusion Criteria:}

All adult patients, ages 18 years old and above, who were referred to the Department of Radiology presenting with SVCO from January 1, 2009 and December 31, 2011 were included in the study.

\section{Exclusion Criteria:}

Patients not included in the study were those who refused to participate or were $<18$ years old. Patients who did not finish the whole course of palliative radiotherapy for SVCO were also excluded from the study.

\section{Sample Procedure:}

Non-probability sampling with inclusion of all patients presenting with Superior Vena Cava Syndrome who received high dose radiotherapy at UP-PGH from 2009 2011.

\section{Maneuvers:}

All patients who were referred to the Department of Radiology who received radiotherapy were accepted provided that they satisfied the inclusion and exclusion criteria. The outcomes of patients who received the SVC dose and the outcomes of patients who completed the entire course of palliative radiotherapy were reviewed. SVC dose received by the patient was defined as 300 cGy fractions $x 4$ doses or 400 cGy fractions x 3 doses, for a total of 1,200 cGy. Depending on the histopathology report, completion radiotherapy was given as 180 or 200 cGy in daily fractions to a total dose of 60 - 66 cGy for primary lung neoplasms and 180 - 200 cGy daily fractions to reach a total dose of 36 to 45 Gy for lymphomas.

Outcomes of patients after receiving palliative radiotherapy were defined as follows:

Recovered - complete resolution of signs and symptoms seen and documented by the physician during the time of referral to the Department of Radiology. These include facial plethora, edema of the head, neck and arms, cyanosis, prominent collaterals, and other relevant clinical findings.

Improved - reduction of signs and symptoms seen and documented by the physician during the time of referral to the Department of Radiology.

Not Improved - no change in the baseline clinical manifestations of the patient as seen and documented by the physician during the time of referral to the Department of Radiology.

Died - permanent termination of the biological functions for more than 48 hours. The possible cause of death of the patient will also be documented.

Lost to follow-up - patients who received initial doses of radiotherapy but were not able to return within 30 days from the last medical check up

\section{General data}

The general data of patients who underwent radiation therapy between January 1, 2009, and December 31, 2011, were taken from files of the Department of Radiation Oncology. The medical records of these patients were retrieved, summarized and analyzed, specifically for diagnosis, symptom(s), duration of presenting symptom, symptomatic relief, and RT dosage (dose/fraction, number of fractions, total dose, and overall treatment time). Histopathologic results were reviewed.

\section{Data Analysis}

Information collected from the patient's records were analyzed using descriptive statistics. Percentages were used to describe and compare the distribution of patients every year. Other qualitative data were illustrated using graphs and figures.

\section{Results}

The Department of Radiology, Section of Radiation Oncology received a total 2,717 referrals from January 1, 2009, to December 31, 2011. These cases included 750 gynecologic malignancies, 600 breast carcinomas, 600 head and neck tumors. The Section was able to treat 137 patients 
( $5 \%$ of total) diagnosed with thoracic malignancies. The rest of the cases were gastrointestinal carcinomas, sarcomas, and metastatic diseases. Of the 137 patients, 75 (55\% of thoracic cases) received palliative treatment for either brain or bone metastasis, 11 ( $8 \%$ of thoracic) underwent definitive chest and mediastinum irradiation, while 51 patients $(37 \%$ of thoracic) underwent high dose radiotherapy for superior vena cava syndrome. Of the 51 patients, only 46 charts were retrieved from the medical records. Of these 46, 38 patients referred for the management of SVCO and whose charts were reviewed were included in the study. Eight patients that were excluded from the study based on the inclusion and exclusion criteria, as follows: five died prior to completing the SVC dose, one was lost to follow up and did not complete the recommended SVC dose/duration and two went home against advice.

The majority of the referrals were charity patients from the general medicine service. One patient underwent radiotherapy twice on the same year due to recurrence of SVCO.

Most (32.5\%) of the patients were $58-67$ years old. The youngest patient was 20 years old and the oldest was 82 years old (Table 1).

Table 1. Demographics

\begin{tabular}{|c|c|}
\hline PARAMETER & CHARACTERISTIC $(n=40)$ \\
\hline Gender (M:F) & $5.7: 1$ \\
\hline \multirow[t]{2}{*}{ Age } & 62 (mean) \\
\hline & $20-82$ (range) \\
\hline \multicolumn{2}{|l|}{ Sources of Referral } \\
\hline Internal Medicine & 18 \\
\hline Pulmonary Medicine & 13 \\
\hline Medical Oncology & 5 \\
\hline Neurology & 1 \\
\hline Nephrology & 1 \\
\hline \multicolumn{2}{|l|}{ Patient Classification } \\
\hline Private & 17 \\
\hline Service & 21 \\
\hline \multicolumn{2}{|l|}{ Clinical Presentation } \\
\hline Dyspnea & 27 \\
\hline Facial Edema & 20 \\
\hline Distended Neck Veins & 13 \\
\hline Upper Extremity Edema & 12 \\
\hline Facial Plethora & 6 \\
\hline Prominent Collateral vessels & 5 \\
\hline \multicolumn{2}{|l|}{ Time Interval from Onset of } \\
\hline \multicolumn{2}{|l|}{ Symptoms to Consult } \\
\hline Less Than 1 week & 3 \\
\hline Two to four weeks & 8 \\
\hline Five to twelve weeks & 3 \\
\hline More than 12 weeks & 4 \\
\hline Undocumented Time Interval & 13 \\
\hline
\end{tabular}

\section{Clinical Symptoms}

Dyspnea was the most common symptom at presentation $(73 \%)$. Other symptoms, in order of frequency, included facial edema, distended neck veins, upper extremity edema, facial plethora and collateral vessels on the anterior chest wall (Table 1).

\section{Duration of Symptoms}

Most of the patients were oblivious to the symptoms until the onset of exertional dyspnea which interfered with their activities of daily living. Three (10\%) patients had symptoms of superior vena cava syndrome for less than 1 week before admission, an additional 8 (28\%) had symptoms of 2 to 4 weeks duration. In 3 patients (10\%), symptoms were present for 5 to 12 weeks and in 4 patients (14\%) for more than 12 weeks. Duration of dyspnea was not documented for 13 patients (Table 1 ).

\section{Diagnostic Methods}

Imaging modalities such as chest $x$-ray and chest CT scan with contrast aided in determining the location and size of lesions. CT scans of 29 patients demonstrated radiographic evidence of extrinsic compression or tumoral infiltration of the superior vena cava.

Histological diagnosis was established either before or after treatment began. Methods employed included either CT scan guided biopsy, ultrasound guided biopsy, bronchoscopy with biopsy, brushing, washing or fine needle aspiration biopsy of accessible lymph nodes. A significant number of patients presented with disseminated disease at diagnosis as evidenced by CT Scan. The most frequent findings in advanced cases were metastasis to mediastinal lymph nodes contralateral to the site of the primary tumor, pleural effusion, and pulmonary nodules separate from the primary mass. Other findings included supraclavicular lymphadenopathies, esophageal involvement, and metastasis to the adrenals, kidneys, pancreas, and bone. Reports revealed that 13 patients $(36.1 \%)$ had non small cell carcinoma particularly adenocarcinoma, 7 patients $(19.4 \%)$ had small cell carcinoma, 1 patient had thymoma. Histological diagnosis was not established in 17 patients $(41.6 \%)$ but 11 of these had results read as "suspicious for malignancy". This non-diagnostic yield is likely due to inadequate sampling especially in cases where only a fine needle aspiration was performed instead of obtaining a tissue sample. Once the section of radiation oncology was informed of these referrals, the patients' history, physical examination, and available laboratory examinations were reviewed. Upon the assessment of SVCO establishing the need for radiotherapy, the proposed procedure was explained to the patient and the relatives. The majority of patients $(77 \%)$ received radiation therapy within three days from referral (Figure 1). Forty patients completed the SVC radiotherapy doses. These patients received a total of 1,200 cGy divided into 300 cGy or 400 cGy fractions. 


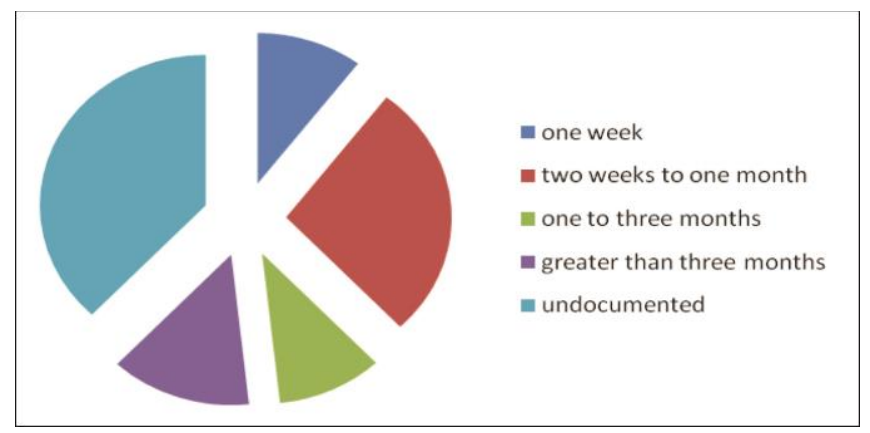

Figure 1. Time interval between onset of symptoms and consult

\section{Symptomatic Response}

Most patients had very good symptomatic response to radiation therapy. The first symptom to be relieved was shortness of breath. At the completion of SVC dose, 2 patients had complete resolution of dyspnea. Twenty-eight of the responders had partial resolution of symptoms, manifested as decreased facial edema and flushing, improvement of dyspnea and decreased anterior chest wall collaterals. Five patients did not experience improvement in any of the baseline symptoms, and three died after completion of the SVC dose (Figure 2).

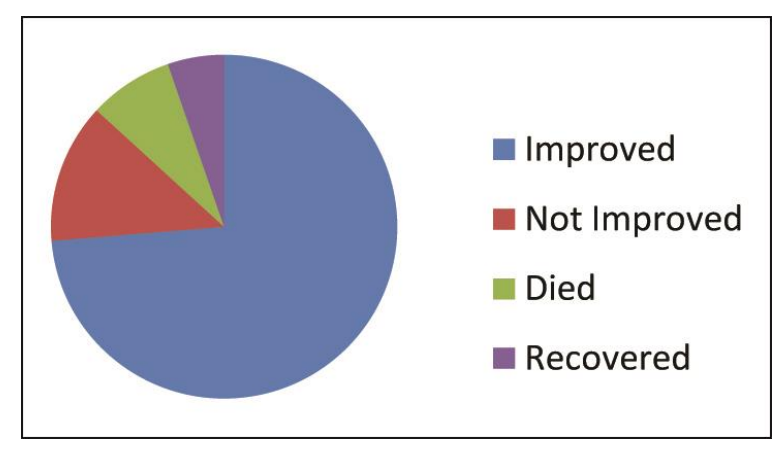

Figure 2. Outcomes of patients treated with SVC doses

Analysis of the outcome of all the responders showed that only eight patients returned for follow up and consented for completion radiotherapy. Two patients died prior to completing the recommended dose. Reported cause of death among these patients was cardiogenic shock. Three patients noted improvement of symptoms but were not able to complete the recommended total dose based on their disease, while one patient's condition worsened medically during the radiation therapy, which prohibited him from completing treatment. Two patients who completed definitive radiotherapy noted further improvement of symptoms.

\section{Discussion}

Radiotherapy is one of the primary treatment modalities for patients with SVCO. The Section of Radiation Oncology received an average of 17 SVCO cases per year since January 1, 2009, to December 31, 2011. The referrals were mostly service patients of the Department of General Medicine and private patients of Adult Pulmonary Medicine.

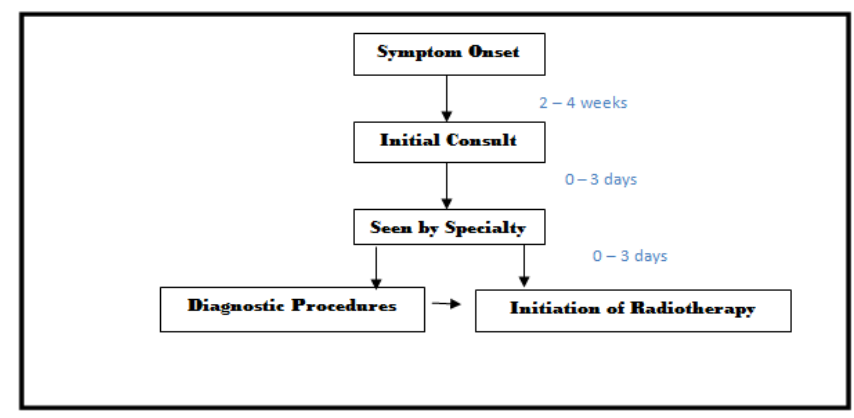

The above presentation summarizes the timeline from the onset of symptoms to initiation of treatment. The subacute presentation of this syndrome is due to the slow and progressive obstruction brought about by the extrinsic compression of the vessel by an enlarging mass. There is a delay of $2-4$ weeks in seeking medical consult in majority of our patients. Though the factors causing these were not investigated, sociodemographic factors such as gender, age, and socioeconomic status may have played a role. Most patients were subsequently scheduled for contrast-enhanced CT scan of the chest. The CT scan served as a guide to the most appropriate procedure in obtaining a histological diagnosis and in planning of radiation treatment fields. $6,15,16$

Malignancy was the major cause of SVCO in our patients. Non small cell lung cancer, particularly adenocarcinoma, accounted for the majority of these cases.

In the Section of Radiation Oncology at UP-PGH, the SVC radiotherapy was given in large initial fractions for rapid palliation of symptoms, according to an observational study conducted by Sy and Benedicto. ${ }^{17}$ SVC radiotherapy is usually given in four 300 cGy fractions or three 400 cGy fractions to reach a total dose of 1,200 cGy. Sy and Benedicto noted that the SVC dose was well tolerated in 23 patients with SVCO. There was a significant reduction in the severity of dyspnea based on the modified Borg score on the third and seventh day of radiotherapy.

After receiving the SVC dose, $72.5 \%$ of patients had improvement of symptoms, especially that of dyspnea. Attending physicians noted a decrease in the severity of facial edema, prominence of collateral vessels on the anterior chest wall, size of the cervical lymphadenopathies, and oxygen requirement. Two patients had complete resolution of symptoms while 5 patients died more than 48 hours after the last dose of radiotherapy. Of the 5 patients, 2 had suspected pulmonary embolism, 1 had cardiogenic shock due to arrhythmia and another patient succumbed to septic 
shock from late-onset hospital-acquired pneumonia. Treatment outcomes of radiotherapy on SVCO were not confirmed among these patients due to overlapping symptoms from the incurred comorbidities. The percentage of service patients who were lost to follow up was $80 \%$. One patient returned after three months due to tumor recurrence and underwent a repeat SVC dose. A significant number of patients did not receive the definitive dose of radiotherapy in both the private $(78 \%)$ and service $(90 \%)$ sectors. These statistics may be due to the patients' underlying conditions. Most of them had extensive disease at diagnosis, frequently found to be stage IV upon complete work-up. As such, radiotherapy might not be the appropriate subsequent treatment option for our patients after the emergency SVC dose, as this modality may not be able to address all sites of metastases. Although the optimal dose and timing of TRT remain controversial, the amount of time from start to completion may affect overall survival.

The importance of a multidisciplinary approach to care from the onset of symptoms, initial consult or admission, diagnostic procedures and treatment cannot be overemphasized. Therapy should be aggressive and patients must be promptly referred to all concerned subspecialties. This will ensure a holistic approach for the management of the individual patient.

\section{Conclusion}

A total of 38 patient profiles were reviewed for SVC syndrome symptoms, etiology, treatment modalities used and treatment outcomes. The majority $(77.5 \%)$ of these cases were due to primary lung malignancies, particularly nonsmall cell lung cancer (64.5\%). Improvement in signs and symptoms, particularly dyspnea, was noted in $72.5 \%$ of patients after receiving high initial dose radiation therapy. Of the 8 patients who followed up for completion therapy, $5 / 8$ of them reported significant improvement.

\section{References}

1. Abner A. Approach to the patient who presents with superior vena cava obstruction. Chest. 1993; 103(4 Suppl):394s-397s.

2. Parish JM, Marschke RF Jr, Dines DE, Lee RE. Etiologic considerations in superior vena cava syndrome. Mayo Clin Proc. 1981; 56(7):407-13.

3. Witt C, Schimdt B, Borges AC, Doerffel W, Baumann G, Romaniuk P. Superior Vena Cava Syndrome. From the Bronchus to the Vessel: A Review. Diagn Ther Endosc. 1997; 4(2):83-93.

4. Armstrong BA, Perez CA, Simpson JR, Hederman MA Role of irradiation in the management of superior vena cava syndrome. Int J Radiat Oncol Biol Phys. 1987; 13(4):531-9.

5. Stanford W, Jolles H, Ell S, Chiu LC. Superior vena cava obstruction: a venographic classification. Am J Roentgenol. 1987; 148(2):259-62.

6. Wilson L, Detterbeck F, Yahalom J. Superior vena cava syndrome with malignant causes. N Engl J Med. 2007; 356(18):1862-9.

7. Urban T, Lebeau B, Chastang C, Leclerc P, Botto MJ, Sauvaget J. Superior vena cava syndrome in small-cell lung cancer. Arch Intern Med. 1993; 153(3):384-7.
8. Wurschmidt F, Bunemann H, Heilmann HP. Small cell lung cancer with and without superior vena cava syndrome: a multivariate analysis of prognostic factors in 408 cases. Int J Radiat Oncol Biol Phys. 1995; 33(1):77-82.

9. Rodrigues $\mathrm{CI}, \mathrm{Njo} \mathrm{KH}$, Karim $\mathrm{AB}$. Hypofractionated radiation therapy in the treatment of superior vena cava syndrome. Lung Cancer. 1993; 10(3-4):221-8.

10. HPS Wai, RMW Yeung, WM Sze, TK Yau, AWM Lee. A review of superior vena cava obstruction in Hong Kong Chinese Patients. Hongkong J Radiol. 2001; 4(2):143-5.

11. Tanigawa N, Sawada S, Mishima K, et al. Clinical outcome of stenting in superior vena cava syndrome associated with malignant tumors: comparison with conventional treatment. Acta Radiol. 1998; 39(6):66974.

12. Doty DB. Bypass of superior vena cava: six years' experience with spiral vein graft for obstruction of superior vena cava due to benign and malignant disease. J Thorac Cardiovasc Surg. 1982; 83(3):326-38.

13. Perez CA, Presant CA, Van Amburg AL III. Management of superior vena cava syndrome. Semin Oncol. 1978; 5(2):123-34.

14. MRC Lung Cancer Working Party. Inoperable non-small cell lung cancer (NSCLC): a Medical Research Council randomised trial of palliative radiotherapy with two fractions or ten fractions. Br J Cancer. 1991; 63(2):265-70.

15. Kim HJ, Kim HS, Chung SH. CT diagnosis of superior vena cava syndrome: importance of collateral vessels. Am J Roentgenol. 1993; 161(3):539-42.

16. Yedlicka JW, Schultz K, Moncada R, Flisak M. CT findings in superior vena cava obstruction. Semin Roentgenol. 1989; 24(2):84-90.

17. Sy TR, Benedicto JP, Sanico-Soliano JP. The effect of superior vena cava dose radiotherapy on dyspnea among patients with superior vena cava syndrome. Chest. 2007; 132(4_MeetingAbstracts):589a. 